\title{
LOS MÁRMOLES DE LA VEGA (BALAZOTE, ALBACETE). TIPOS Y MORFOLOGÍA
}

\author{
JULIA SARABIA BAUTISTA \\ F. JAVIER MUÑOZ OJEDA \\ Universidad de Alicante
}

\begin{abstract}
Las excavaciones en La Vega de Balazote (Albacete), proporcionaron, a finales de la década de los ochenta, uno de los conjuntos marmóreos más importantes del panorama rural hispano de finales del Imperio. En este trabajo presentamos los diferentes tipos aparecidos y su posible adscripción cronológica y espacial dentro del grupo de edificaciones halladas en el yacimiento, pertenecientes en su mayoría a opera sectilia parietales y pavimentales.

At the end of the nineteen-eighties, the excavations in the Vega of Balazote (Albacete) provided one of the most important collections of marbles in the Hispanic rural world at the end of the Roman Empire.

In this paper we look at different types of marble that have been found in the Vega of Balazote, and their possible chronology and connexion with the buildings found on the site, most of which are mosaics, wall facings and pavements.
\end{abstract}

El conjunto de materiales marmóreos que se estudia en este trabajo representa un ejemplo único en el Conuentus Carthaginensis de la ejecución de grandes programas ornamentales en ambientes domésticos rurales del Bajo Imperio, ya que, a diferencia de lo que se suele pensar para los procesos arquitectónicos públicos de esta época, en La Vega de Balazote (Albacete) se edificaron suntuosos espacios de vivienda en los que se emplearon grandes cantidades y numerosos tipos de placas marmóreas que revistieron las paredes y suelos de algunos de esos espacios a modo de opera sectilia.

Estos ambientes arquitectónicos fueron hallados en una excavación de salvamento llevada a cabo en 1986 por J. L. Frías con motivo de la construcción de un polígono industrial en el término municipal de Balazote. Los solares estaban localizados a un kilómetro al noroeste de la conocida como Villa de Balazote (Camino Viejo de las Sepulturas), donde en los años 70 se documentaron importantes restos arquitectónicos de época romana pertenecientes a un complejo residencial de envergadura. La proximidad del futuro polígono industrial a dichas estructuras hacía prever la existencia de nuevos restos relacionados con tal complejo doméstico, de ahí que los excavadores plantearan una actuación en tres frentes o sectores -A, B y C-que permitieran el mayor conocimiento de los posibles hallazgos.

Tradicionalmente ${ }^{1}$, se ha considerado que las estructuras halladas en esta excavación de La Vega de Balazote (Fig. 1), formaron parte de la zona rústica de la conocida villa hallada en el Camino Viejo de las Sepulturas ${ }^{2}$. Dichas estructuras configuraban un conjunto de estancias de pequeñas dimensiones que fueron interpretadas como almacenes y quizá talleres y casas de los siervos o empleados de la villa.

Una de las estancias más interesantes, localizada en el Sector $C$, era la que se definió como un patio o espacio abierto, de planta rectangular, con una extensión aproximada de

\footnotetext{
1. Meseguer, 1989, 1119-1127 y Sanz, 1995, 345 ss.

2 En este artículo únicamente nos proponemos presentar los tipos, porcentajes de aparición y posible ubicación arquitectónica de los mármoles aparecidos en La Vega, de ahí que no hagamos un análisis detallado de la pertenencia o no de estas estructuras rurales a los ambientes rústicos de la villa del Camino Viejo de las Sepulturas. El estudio de estos dos yacimientos y su conexión e interpretación forman parte de la tesis doctoral de Julia Sarabia, cuyos resultados serán defendidos a corto plazo.
} 


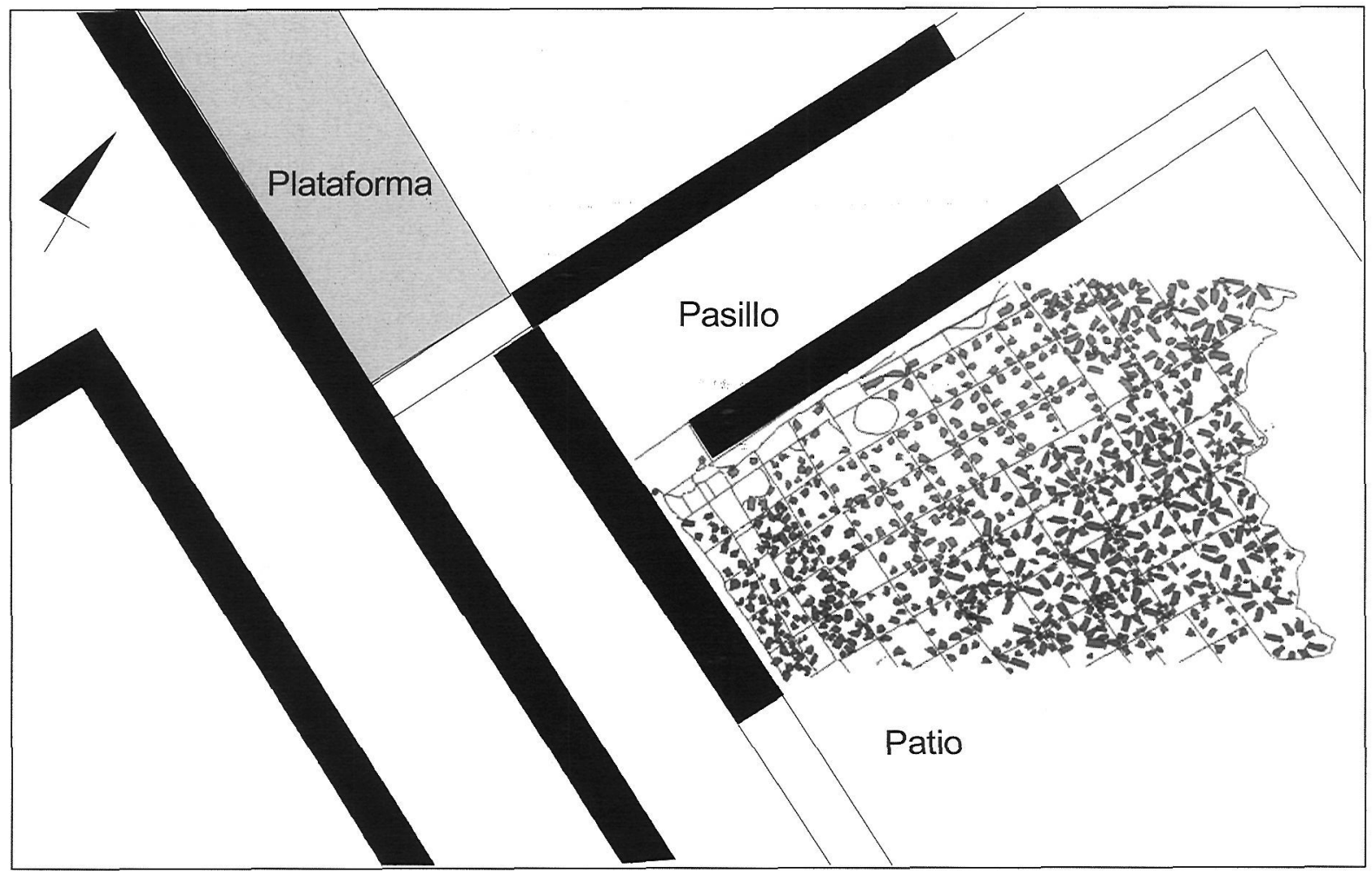

Figura 1: Planta esquematizada de las estructuras halladas en el Sector C de La Vega ${ }^{3}$. En el patio se halló parte de un pavimento de mortero con crustae cerámicas.

$20 \mathrm{~m}^{2}$, con acceso a un corredor o pasillo que lo rodeaba en todo el perímetro descubierto. Tras excavar los niveles de derrumbe del patio, formados por una gran cantidad de ímbrices, se halló una acumulación de mármoles de diversos tipos y procedencias con un peso aproximado de $500 \mathrm{~kg}$. La mayoría de estos mármoles presentaban formas de lastras geométricas, propias de los opera sectilia, formando un conjunto de gran interés para su estudio, de ahí que lo presentemos en este trabajo ${ }^{4}$.

\section{TIPOS DE MÁRMOLES DOCUMENTADOS EN LA VEGA ${ }^{5}$}

Al observar la gráfica expuesta a continuación (Fig. 2), vemos cómo destacan claramente cuatro tipos de marmora: cipollino, giallo antico ${ }^{6}$, pavonazzetto y greco scritto, sobre las demás clases identificadas: serpentino, porfido rosso, caliza alabastrina, africano, portasanta, lumachella, rosso antico y breccia corallina. En cuanto a los diferentes tipos de mármol blanco, aunque sabemos que sin un riguroso análisis químico de sus componentes no podemos aventurar la procedencia de muchos de ellos, parece clara la predominante presencia de proconnesso, lo cual debemos destacar por su importancia a la hora de hablar tanto de la procedencia del conjunto marmóreo como de su cronología.

Un dato a destacar es el grosor que presentan las piezas estudiadas, en su mayoría placas de revestimiento, con una media que oscila entre 1 y 3 centímetros. La importancia de este parámetro radica en que está estrechamente relacionado con el tipo de mármol empleado; así un mármol de importación llegará a la península con toda probabilidad

\footnotetext{
${ }^{3}$ Existen algunas planimetrías que nos permiten definir los espacios excavados, pero todas ellas muy esquematizadas. Faltan en muchos de los casos la representación de los vanos de acceso y otro tipo de estructuras, por lo que no podemos presentar planimetrías detalladas, sólo croquis.

${ }^{4}$ Existen dos trabajos anteriores sobre el estudio de estos mármoles, que recogen algunos aspectos arqueológicos y analíticos del conjunto pero sin adentrarse en un análisis exhaustivo de los tipos y porcentajes aparecidos: Meseguer, 1989, 1119-1127 y Arana, 1989, 869-876.

5 Queremos agradecer a Rubí Sanz y a Blanca Gamo (Museo Arqueológico Provincial de Albacete) la ayuda prestada en la catalogación e inventariado del material marmóreo. Además, hacemos extensivo este agradecimiento a Begoña Soler (Universidad de Murcia) por sus apreciaciones en el reconocimiento de algunos de los tipos de mármol estudiados.

${ }^{6}$ En sus distintas variedades, es decir, brecciato y dorato.
} 


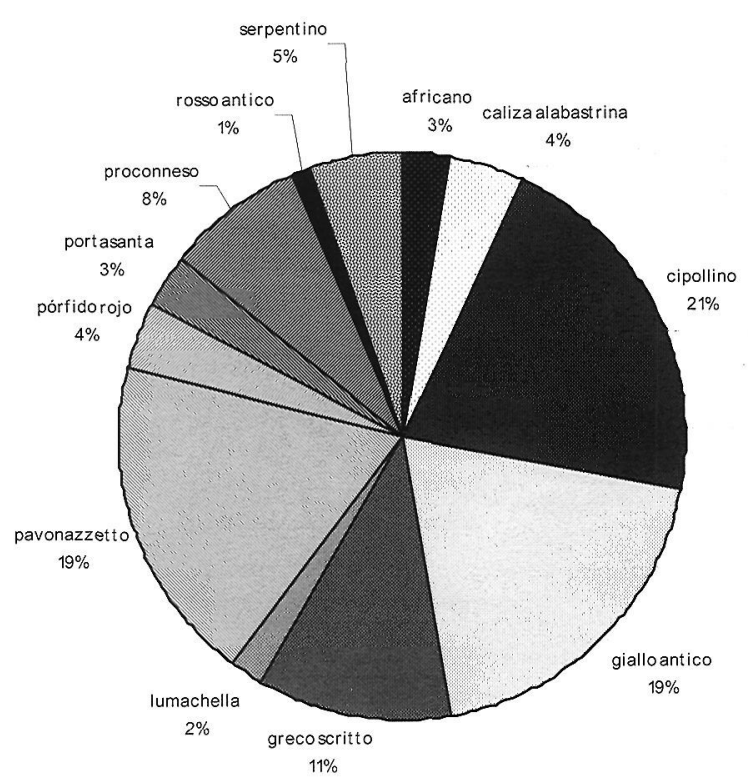

Figura 2: Gráfica con los porcentajes de los distintos tipos de marmora aparecidos en La Vega.

presentando un tallaje más fino que un mármol autócto, abaratándose así los costes de transporte: a mayor cargamento mayor rentabilidad. Sobresalen de esta media dos fragmentos, una placa de cipollino $(7 \mathrm{~cm})$ y una placa moldurada de proconesso $(3 \mathrm{~cm})$, cuyo mayor grosor podría deberse a un reempleo de estos soportes que en origen no debieron haber funcionado como placas de revestimiento parietal o pavimental, quizá columnas, lo que les confirió un volumen diverso.

\section{ANÁLISIS DE LOS TIPOS MÁS DES- TACADOS}

\section{Cipollino (Fig. 3)}

Las canteras de cipollino se sitúan a lo largo de al menos cinco distritos: Karystos, Marmarion, Styra, Animborio y Karatza, en la costa meridional de la isla griega de Eubea. Este mármol se usa normalmente para la realización de columnas, tal como demuestran los restos encontrados tanto a pie de cantera (Pensabene, 1994, 254-272) como en la statio marmórea de Ostia (Pensabene, 1994, 85-98). Su empleo está testimoniado desde inicios del siglo I d.C., pero su momento de máxima explotación se sitúa a finales del siglo I d.C., utilizándose de forma profusa para la realización de las columnas del foro de Trajano. La importación de este mármol se realiza en columnas (ya sean totalmente elaboradas o semielaboradas) o en grandes bloques trapezoidales y no en placas, tal como encontramos en el conjunto estudiado. Ésto nos debe hacer reflexionar sobre el lugar de procedencia. Independientemente de que hayan llegado a su lugar de destino elaboradas o semielaboradas, lo que sí es seguro es que han sido extraídas de bloques o columnas acumuladas en stock en una statio o taller especializado en la elaboración de este tipo de placas. Dependiendo de la cronología del conjunto estudiado, el lugar en el que se tallaron estas placas variará, así por ejemplo, si nos encontrásemos ante un conjunto datado en el siglo I d.C. seguramente tendríamos que remitirnos a una obra realizada por un taller ostiense, mientras que para un enclave edificado en época bajoimperial la procedencia artesanal más lógica sería norteafricana o de tradición norteafricana ${ }^{7}$.

\section{Giallo antico (Fig. 4)}

Las canteras de este mármol se sitúan en Chemtou (actual Túnez), antigua región de Numidia, de ahí el nombre de marmor numidico. Su uso está atestiguado desde época muy temprana. Plinio llama la atención sobre su primer uso en Roma ${ }^{8}$ por lo peculiar de encontrar mármol de color en la arquitectura privada; el mismo autor también describe cómo M. Lepidus (cónsul en el 78 a.C.) puso un umbral de este mármol en su casa. Al contrario que el cipollino, el giallo antico tiene un empleo más prolífico debido a sus variedades, que lo hace más versátil. En su variedad dorada, con un corte muy próximo al alabastro, se usa normalmente para la realización de finas placas utilizadas en sectilia, en scutulata, etc.; la calidad brecciata, de un aspecto más sólido, es empleada tanto para columnas como para escultura. Gracias al estudio de las marcas epigráficas sobre este mármol (Dubois, 1908), se conoce perfectamente en qué manos ha estado la propiedad de las canteras numídicas ${ }^{9}$.

\footnotetext{
${ }^{7}$ La fecha bajoimperial de las estructuras en que aparecen estos materiales, estudiados por J. Sarabia en su tesis doctoral, apunta más bien hacia la segunda de estas hipótesis.

${ }^{8}$ Plinio, N.H., XXXVI, 7, donde describe el uso por parte de L. Licinius Crassus de columnas de marmor numidicum.

${ }^{9}$ Dubois, (1908, 39-45). Tras la adhesión de Numidia, Agripa se hace cargo de la explotación de las canteras, y a su muerte la propiedad pasa a manos de la casa imperial; los epigrafes más tardíos sobre bloques extraídos son de época de Marco Aurelio y Lucio Vero.
} 
Aquí nos encontramos ante un hecho similar al del cipollino. Observamos, dentro del conjunto de placas estudiadas, todas las variedades de giallo antico, lo que nos hace pensar que procedan de un lugar en el que se encuentren almacenados los distintos tipos usados, ya sea de una statio o de talleres situados muy próximos a la cantera.

\section{Pavonazzetto (Fig. 5)}

El conocido como mármol synnadico tiene su lugar de origen en Tchifout-Kassaba e Itschki-Karahissa (Frigia), de donde además se extrae una variedad de mármol blanco (Docimium). Al valor estético de este tipo de mármol se le suma un valor simbólico debido a su lugar de procedencia. Tras el establecimiento de la pax por parte de Augusto $^{10} \mathrm{y}$ la anexión de Frigia a Roma, el princeps mandará construir para su foro en Roma un trípode sustentado por tres esculturas realizadas en pavonazzetto, representando a tres prisioneros partos (De Nuccio, 2002, 425-430). Esta irónica representación será repetida por Trajano para su foro, donde construirá a modo de atlantes una serie de prisioneros dacios y partos en mármol synnadico. Este último momento coincide con el del auge de la explotación de estas canteras. Es una piedra de gran polivalencia, usada para lastras parietales y pavimentales ${ }^{11}$, columnas, escultura y mobiliario (urnas, trapezoforos, etc.).

En cuanto a la posesión de las canteras, no parece tan claro que, como ocurre en Chemtou, la propiedad recaiga plenamente sobre la casa imperial; de hecho no aparecen marcas con nombre del emperador sobre bloques de pavonazzetto hasta época de Trajano. Es en este momento, como hemos señalado anteriormente, cuando se incrementa la explotación de las canteras, por lo que hemos de suponer un mayor control de las mismas, finalizando su explotación hacia la época de los Antoninos. Si atendemos a la acumulación de pavonazzetto en la statio de Ostia, vemos que hay una clara superioridad de bloques frente a columnas ${ }^{12}$, lo que indica la preferencia de este mármol para la elaboración de piezas reducidas, como es el caso de placas de revestimiento parietal o pavimental.

\section{Greco scritto (Fig. 6)}

Este mármol de procedencia norteafricana no está atestiguado en Roma hasta inicios del siglo II d.C. (Pesabene, 1976, 185), momento en el que empieza a cesar la explotación del bardiglio ${ }^{13}$, de aspecto muy parecido al greco scritto. Tal vez este cambio esté en directa relación con la importación a las provincias occidentales de este tipo de mármol, en detrimento de la piedra italiana. Su uso en Ippona, localidad donde se encuentran las canteras del greco scritto (canteras de Cap de Garde), se atestigua desde finales del siglo I a.C. (Pesabene, 1976, 181), como piedra para sillería y no como soporte para la ornamentación arquitectónica.

El empleo de este mármol suele estar destinado a la elaboración de piezas de pequeño y mediano tamaño, es decir, capiteles, lastras parietales, pavimentales, etc. Pensabene, en su estudio sobre el uso del greco scritto (Pensabene, 1976) en Ippona, habla de una propiedad privada para las canteras de Cap de Garde, lo que sin duda se ve reforzado por la no presencia de este mármol en la statio de Ostia. Este hecho nos puede hacer pensar en un comercio directo entre la cantera, o los talleres que se abastecen de ésta, y el centro demandante, sin necesidad de tener que pasar por el control administrativo de Roma, lo que se da ya en pleno siglo II d.C. También habria que subrayar el uso de este mármol preferentemente para la decoración en ámbitos privados, lo que estaría en relación directa con la propiedad privada de las canteras.

\section{Proconneso (Fig. 7)}

A pesar de no encontrarse entre los tipos más abundantes, vamos a detenernos en este mármol blanco de procedencia oriental por lo peculiar de su uso. Tradicionalmente, a este mármol se le había asociado un uso local o una difusión restringida a las provincias orientales, con cronologías tardoantiguas y protobizantinas (Dubois, 1908, 96) ${ }^{14}$. Pero J. B. Ward-Perkins

\footnotetext{
${ }^{10}$ Pensabene $(1998,8)$. Aunque ya se constata su uso en época tardorrepublicana, en lastras parietales y pavimentales del Templo de la Concordia y la Basílica Julia.

11 Gnoli señala que suele combinarse con Giallo Antico y Africano (Gnoli, 1988, 144).

12 Pensabene (1994, 422-423). De un total de 26 piezas localizadas en la statio de Ostia, 4 son columnas y 21 son bloques trapezoidales.

13 Variante del mármol extraído en Carrara que combina el. color blanco y azul, al igual que el mármol descrito.

${ }^{14} \mathrm{Sin}$ hacer referencia a ninguna marca epigráfica sobre este tipo de mármol.
} 
(Ward-Perkins, 1992, 37) propone un modelo para la distribución de determinados tipos de mármol en las provincias, reflexionando de forma especial sobre el proconneso y el mármol griego como los preferidos para la realización de sarcófagos y su redistribución por las provincias norteafricanas. Este despegue del proconneso se da a inicios del siglo II d.C., aunque su uso se atestigua desde los primeros años del principado de Augusto. Las reflexiones sobre el empleo y distribución de mármoles blancos orientales manufacturados, a través de agencias ultramarinas, entre la cantera y el centro demandante ya lo adelantó J. C. Fant (Fant, 1993); J. B. Ward-Perkins partiendo de esta idea, analizará en la Tripolitana cómo estas agencias terminan acaparando la ordenación y distribución de los programas decorativos de forma íntegra, dejando de ser así simples intermediarios (Ward-Perkins, 1992, 63). Los últimos estudios en la Tripolitana (Pensabene, 2001), en concreto sobre las dos ciudades más notables de la provincia proconsular (Sabratha y Leptis Magna), demuestran un aporte de mano de obra oriental, seguramente por parte de estas agencias, para la elaboración de los programas decorativos tripolitanos.

La presencia de este tipo de mármol en provincias occidentales, ya sea manufacturado a modo de sarcófago o en placas parietalespavimentales, como en nuestro caso, nos hace plantear cuestiones sobre el lugar de procedencia del material marmóreo. Como ya hemos visto más arriba, desde el siglo II d.C. existen talleres en pleno funcionamiento en la Tripolitana, en disposición no sólo de distribuir el material para la realización de programas ornamentales a nivel local, sino también de exportarlos. Puesto que cuentan tanto con mano de obra especializada, como de materia prima y del mecanismo redistribuidor (las agencias de Ward-Perkins), parece posible proponer una relación directa entre talleres norteafricanos ${ }^{15}$ y centros demandantes hispanos.

\section{CONCLUSIONES}

Si recapitulamos acerca de los datos obtenidos en el estudio de este conjunto marmóreo, podemos plantear dos conclusiones preliminares en cuanto a la cronología y la factura del mismo.

Ciertos tipos de mármol nos ofrecen un marco cronológico en lo que a su uso se refiere.
Un claro ejemplo es el greco scritto, cuyo empleo no se detecta antes del siglo II d.C. fuera de su lugar de explotación. Ciñéndonos a la aparición de este tipo dentro del conjunto de La Vega, podemos decir que el programa decorativo en el que se incluye, no puede realizarse antes de esa fecha. Partiendo de esta premisa, podemos asignar, de una manera coherente, los otros tipos aparecidos a esta cronología. Así por ejemplo, la presencia de cipollino se podría vincular con su segundo gran momento de explotación, durante el inicio del siglo II d.C.

Pero sin duda alguna, es la vinculación entre proconneso y siglo II d.C. Io que nos permite hablar de la procedencia y elaboración del conjunto. Como sabemos, el uso del proconneso se generaliza desde el siglo II d.C en detrimento de la variedad blanca de Carrara, creciendo su empleo junto al Pentélico y otras variedades micro-asiáticas. La manufacturación del proconneso (en realidad cualquier tipo de mármol blanco) precisa de artesanos altamente especializados, lo que se documenta de forma clara para estas fechas en el norte de África. En las provincias norteafricanas, se detecta durante este siglo, el auge económico que favorece a sus élites locales. Este despegue provoca una gran demanda de programas decorativos, tanto públicos (ciudades como Leptis Magna o Sabratha experimentan en estos momentos una total trasformación), como privados (aparición de las grandes villae norteafricanas). Esta creciente demanda ornamental favorece probablemente la creación, a medio y largo plazo, de una statio de similares características a la de Roma, lo que hará posible que las agencies actúen de una forma autónoma respecto a Roma, desvinculándose asi del control administrativo que suponia adquirir el mármol en Ostia. La creación de este circuito directo entre las canteras orientales y el norte de África se prolongará hacia occidente cuando desde Hispania se desarrollen programas decorativos de los talleres norteafricanos. Éste es el caso que nos ocupa, un conjunto de mármol perteneciente a un programa decorativo

\footnotetext{
15 Pensabene $(1994,422-423)$. Descartamos una procedencia ostiense por la escasa presencia de este mármol en la statio marmorum de Ostia (4 piezas). De igual modo la escasez de epigrafia sobre esta variante de mármol nos debe hacer reflexionar sobre la autonomía de este tipo de mármo al margen de la administración de la statio marmorum de Ostia.
} 
contemporáneo que probablemente provenga de estos talleres norteafricanos. Así, su llegada a la península Ibérica se desmarca de los circuitos tradicionales de época altoimperial, se adscribe a la nueva vía Oriente-Norte de ÁfricaOccidente.

La aparición de un mármol de sustitución local, como es el caso del greco scritto, junto a la gran cantidad de mármoles orientales que aparece en el conjunto estudiado, es lo que nos sugiere una procedencia norteafricana. La aparición del mármol oriental en lugar del de Carrara no hace más que reforzar la idea de una procedencia no itálica. En caso de que procediese de un taller situado en Ostia, habríamos detectado una presencia de mármol blanco itálico, abundante en la statio ostiense.

Julia Sarabia Bautista

c/ Deportista Ramón

Mendizábal, $27,4^{\circ} \mathrm{lzda}$.

03006 Alicante

julia.sarabia@ua.es

\section{BIBLIOGRAFÍA}

ARANA, R. 1989: "Estudio de los mármoles del yacimiento de "La Vega", Balazote (Albacete). II. Aspectos Mineralógicos", XIX Congreso Nacional de Arqueología, Zaragoza, 869-876.

COOPER, F. 1981: "A source of Ancient Mable in the Southern Peloponnese", AJA, 85, 190-191.

DE NUCCIO, M. 2002: "Storia e restauri dei Barbari inginocchiati", I marmi colorati della Roma imperiale, Mercati di Traiano (28 settembre 2002 - 19 gennaio 2003), 425-430.

DUBOIS, Ch. 1908: Étude surl'administration et l'exploitation des carriéres dans le monde romain, Paris.

FANT, J.C. 1993: "The Roman Imperial Marble Trade: a distribution Model", en R. Francovich, Archeologia della attività strattive e metallurgiche, 89-92, Florencia.
GAGGIOTTI, M. 1987: "L'importazione del marmo numidico a Roma in epoca tardorrepubblicana, L'Africa Romana", Atti del IV Convegno di Studio, Sassari 12-14 Dicembre 1986, 201-213, Sassari.

GNOLI, R. 1988: Marmora romana, Roma.

HANKEY, V. 1965: "A marble Quarry at Karystos", BmusBeyrouth, 18, 53-59.

LAZZARINI, L. 1990: "Rosso Antico and other Red Marbles used in Antiquity: A Charaterization Study", en AAVV, Marble. Art Historical and Scientific Perspectives on Ancient Sculpture (Papers delivered at a Symposium at the J. Paul Getty Museum, April 28-30, 1988), 237-252, Malibu.

MESEGUER, M. S. 1989: "Estudio de los mármoles del yacimiento de "La Vega", Balazote (Albacete). I. Aspectos Arqueológicos", XIX Congreso Nacional de Arqueología, 1119-1127, Zaragoza.

MONNA, D. y Pensabene, P. 1977: Marmi dell'Asia Minore, Roma.

PENSABENE, P. 1976: “Sull'impiego del marmo di Cap de Garde. Condizioni giuridiche e significato economico delle cave in etá imperiale", In memoria de Giovanni Becatti, Studi Miscellanei n. 22, 177-190, Roma.

PENSABENE, P. 1994: Le Vie del marmo. I blocchi di cava di Roma e di Ostia: Il fenomeno del Marmo nella Roma Antica, Itinerari Ostiensi, VII, Roma.

PENSABENE, P. 1998: /l marmo e il colore. Guida fotografica I marmi della collezione Podesti, en Marmi Antichi II (P. Pensabene y Matthias Bruno), Roma.

PENSABENE, P. 2001: "Pentelico e proconnesio in Tripolitania: coordinamento o concorrenza nella distribuzione? Contributo ad una nuova discussione dei modelli di Ward Perkins sulla circolazione del marmo", ArchCl, 52, 63-127.

RÖDER, J. 1971: "Marmor Phrygium. Die antiken Marmorbrüche von Iscehisar in Westanatolien", Jdl, 86, 253312.

SANZ, R. 1995: "El poblamiento rural del área de Balazote (Albacete) a la luz de las últimas investigaciones", Poblamiento rural romano en el Sureste de Hispania (Actas de las Jornadas celebradas en Jumilla del 8 al 11 de noviembre de 1993), 345 ss, Murcia.

WARD-PERKINS, J. B. 1951: "Tripolinaia and the Marble Trade", JRS, 41, 89-104.

WARD-PERKINS, J. B. 1992: Marble in Antiquity, Collected Papers, London. 


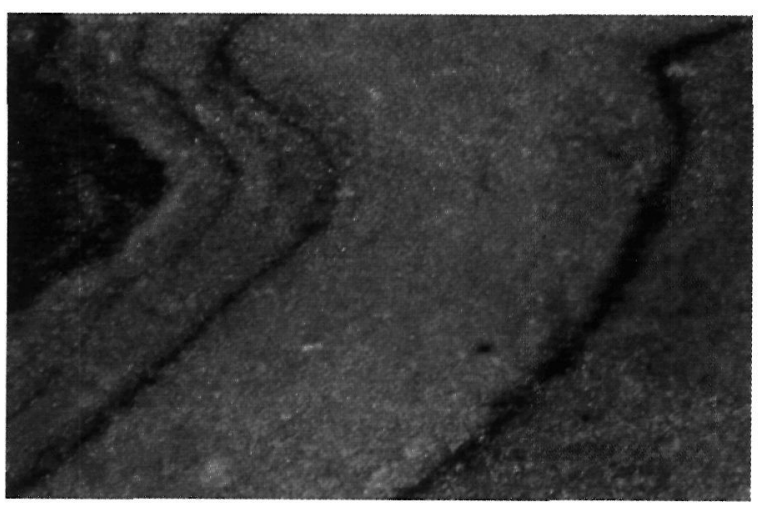

Figura 3: Muestra de una de las placas de cipollino halladas en La Vega de Balazote.

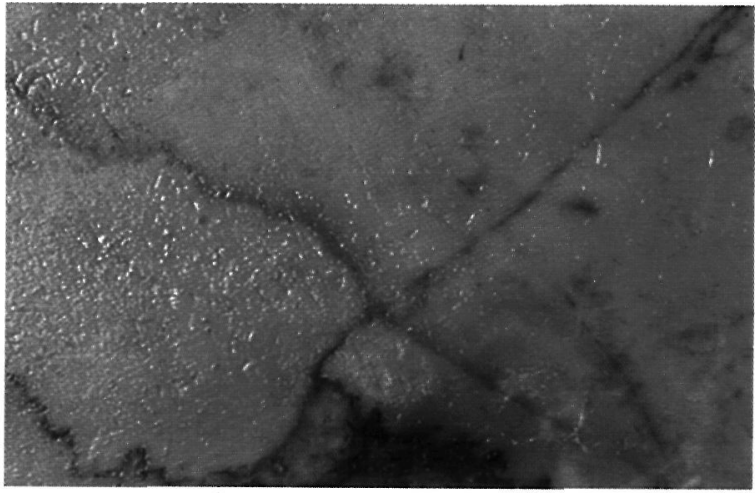

Figura 4: Muestra de giallo antico perteneciente a una placa del conjunto estudiado.

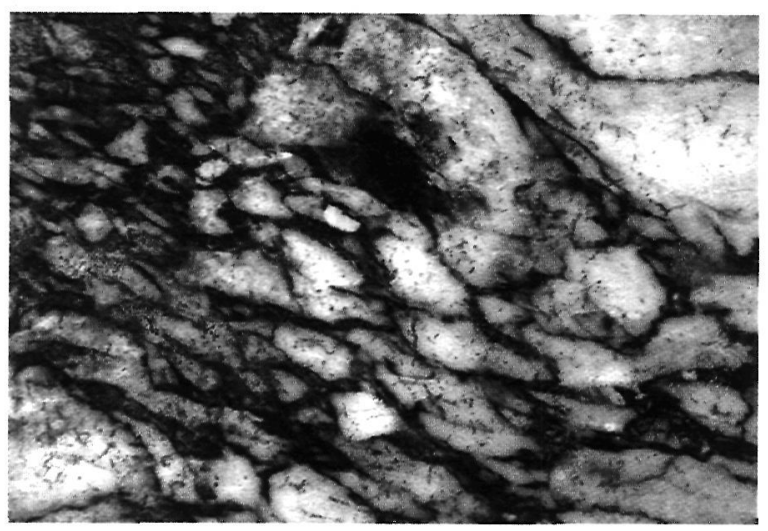

Figura 5: Ejemplo de una de las numerosas placas de pavonazzetto halladas.

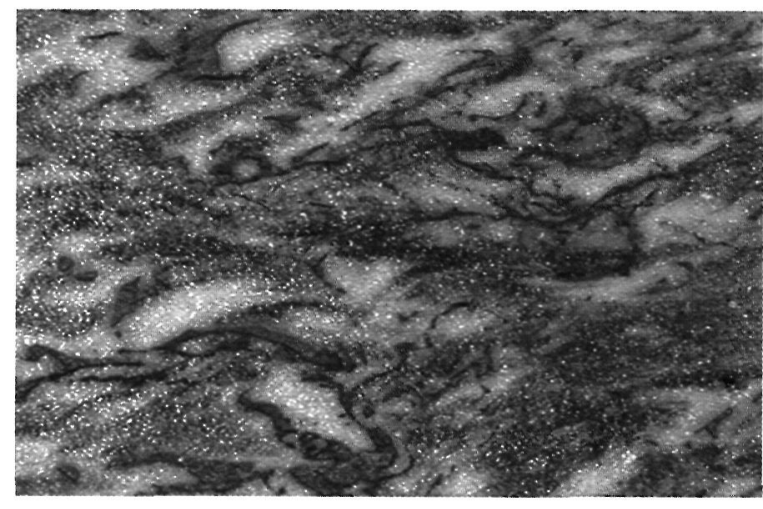

Figura 6: Detalle de la variante de greco scritto aparecida en La Vega.

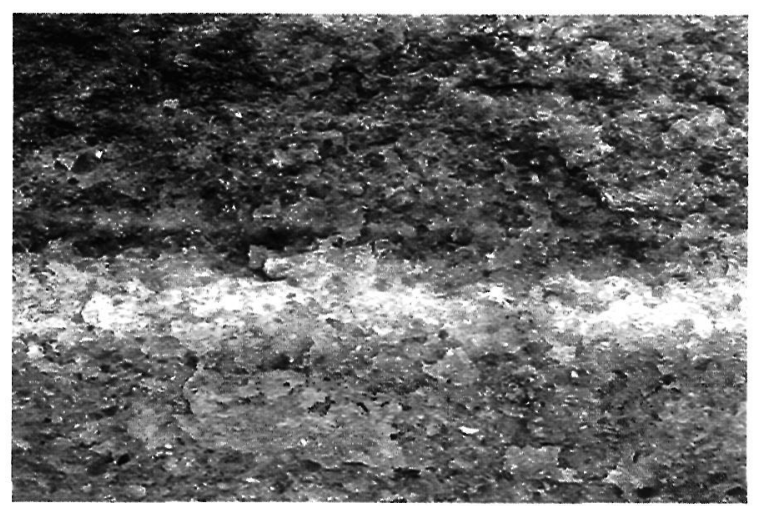

Figura 7: Vista de los componentes del mármol proconneso del enclave. 